\title{
Exome Sequencing in 200 Intellectual Disability/Autistic Patients: New Candidates and Atypical Presentations
}

\author{
Floriana Valentino ${ }^{1,2,+}$, Lucia Pia Bruno ${ }^{1,2,+}$, Gabriella Doddato ${ }^{1,2}$, Annarita Giliberti ${ }^{1,2}$, Rossella Tita ${ }^{3}$, \\ Sara Resciniti 1,2, Chiara Fallerini 1,2 ${ }^{1}$, Mirella Bruttini 1,3, Caterina Lo Rizzo ${ }^{3}$, Maria Antonietta Mencarelli ${ }^{3}$, \\ Francesca Mari 1,2,3 (D), Anna Maria Pinto ${ }^{3}$, Francesca Fava 1,2,3 (D), Margherita Baldassarri 1,2 (D), \\ Alessandra Fabbiani 1,2,3, Vittoria Lamacchia 1,2,3, Elisa Benetti ${ }^{2}$, Kristina Zguro ${ }^{2}$, Simone Furini ${ }^{2}$, \\ Alessandra Renieri 1,2,3 and Francesca Ariani 1,2,3,*
}

check for updates

Citation: Valentino, F.; Bruno, L.P.; Doddato, G.; Giliberti, A.; Tita, R.; Resciniti, S.; Fallerini, C.; Bruttini, M.; Lo Rizzo, C.; Mencarelli, M.A.; et al. Exome Sequencing in 200 Intellectual Disability/Autistic Patients: New Candidates and Atypical Presentations. Brain Sci. 2021, 11, 936. https://doi.org/10.3390/ brainsci11070936

Academic Editor: Corrado Romano

Received: 10 June 2021

Accepted: 13 July 2021

Published: 16 July 2021

Publisher's Note: MDPI stays neutral with regard to jurisdictional claims in published maps and institutional affiliations.

Copyright: (c) 2021 by the authors. Licensee MDPI, Basel, Switzerland. This article is an open access article distributed under the terms and conditions of the Creative Commons Attribution (CC BY) license (https:/ / creativecommons.org/licenses/by/ $4.0 /)$.
1 Medical Genetics, University of Siena, 53100 Siena, Italy; floriana.valentino@dbm.unisi.it (F.V.); lucia.bruno@dbm.unisi.it (L.P.B.); gabriella.doddato@dbm.unisi.it (G.D.); giliberti@student.unisi.it (A.G.); sara.resciniti@dbm.unisi.it (S.R.); fallerini2@unisi.it (C.F.); mirella.bruttini@dbm.unisi.it (M.B.); francesca.mari@dbm.unisi.it (F.M.); francesca.fava@dbm.unisi.it (F.F.); margherita.baldassarri@dbm.unisi.it (M.B.); alessandra.fabbiani@dbm.unisi.it (A.F.); vittoria.lamacchia@dbm.unisi.it (V.L.); alessandra.renieri@dbm.unisi.it (A.R.)

2 Med Biotech Hub and Competence Center, Department of Medical Biotechnologies, University of Siena, 53100 Siena, Italy; elisa.benetti@dbm.unisi.it (E.B.); kristina.zguro@student.unisi.it (K.Z.); simone.furini@dbm.unisi.it (S.F.)

3 Genetica Medica, Azienda Ospedaliera Universitaria Senese, 53100 Siena, Italy; rossella.tita@dbm.unisi.it (R.T.); lorizzo2@unisi.it (C.L.R.); mariaantonietta.mencarelli@unisi.it (M.A.M.) annamaria.pinto@dbm.unisi.it (A.M.P.)

* Correspondence: francesca.ariani@unisi.it; Tel.: +39-0577-233-303; Fax: +39-0577-233-325

+ Co-first authors.

Abstract: Intellectual disability (ID) and autism spectrum disorder (ASD) belong to neurodevelopmental disorders and occur in $\sim 1 \%$ of the general population. Due to disease heterogeneity, identifying the etiology of ID and ASD remains challenging. Exome sequencing (ES) offers the opportunity to rapidly identify variants associated with these two entities that often co-exist. Here, we performed ES in a cohort of 200 patients: 84 with isolated ID and 116 with ID and ASD. We identified 41 pathogenic variants with a detection rate of $22 \%(43 / 200): 39 \%$ in ID patients (33/84) and $9 \%$ in ID/ASD patients (10/116). Most of the causative genes are genes responsible for wellestablished genetic syndromes that have not been recognized for atypical phenotypic presentations. Two genes emerged as new candidates: CACNA2D1 and GPR14. In conclusion, this study reinforces the importance of ES in the diagnosis of ID/ASD and underlines that "reverse phenotyping" is fundamental to enlarge the phenotypic spectra associated with specific genes.

Keywords: exome sequencing; intellectual disability; autism spectrum disorder

\section{Introduction}

Intellectual disability (ID) is characterised by significant limitations in intellectual functioning (reasoning, learning, problem-solving) and adaptive behaviour (conceptual, social, and practical skills) that originate before the age of 18 [1]. Affecting $1-3 \%$ of the world's population, ID represents an important socio-economic problem in healthcare [2,3]. ID is characterized by limitations in cognitive functions that manifest as an intelligence quotient (IQ) below 70. ID may be "isolated" or "syndromic" when patients have peculiar facies, specific physical signs and/or an abnormal growth pattern [4].

Autism spectrum disorder (ASD) is characterized by deficient social interactions, poor or absent communication, repetitive behaviours, and apparently limited interests [5]. ASD generally becomes apparent after the first year of life and it has been reported in an increasing number (2.2-2.7\%) of children, with boys four times more likely to be affected 
than girls [6,7]. From a clinical point of view, the autism spectrum disorder is subdivided into "syndromic" when a co-occurrence between autism (ASD) and dysmorphic features, also other somatic or neurobehavioral abnormalities could be observed [8].

ID and ASD often co-exist and identifying the etiology of the two conditions remains challenging due to disease heterogeneity. Accurate clinical, as well as molecular diagnoses are essential for a deeper understanding of the pathogenesis of these conditions and for devising tailored treatments [4]. Until the advent of the first next generation sequencing (NGS) platforms, a large fraction of cases remained not diagnosed, with many families undergoing a "diagnostic odyssey" [9]. The introduction of exome or genome sequencing (ES/GS) has significantly improved diagnostic rates in individuals with suspected ID/ASD genetic disorders refractory to conventional diagnostic testing [10].

In the present study, ES was generated for a total of 200 individuals (84 ID and 116 ID/ASD patients). Pathogenic or likely pathogenic (P/LP) variants were found in 43 individuals $(22 \%)$, with 45 variants of uncertain significance in an additional $20 \%$ (40/200). Our data strongly support the value of large-scale sequencing, especially ES within proband-parent trios, as an effective first-choice diagnostic tool.

\section{Materials and Methods}

\subsection{Selection of Patients and DNA Samples' Preparation}

Genetic counselling was carried out to evaluate each patient's personal and familial history. Parents provided and signed a written informed consent at the Medical Genetics department of the University of Siena, Italy, for exome sequencing analysis, clinical data usage, and the use of DNA samples from the tested individuals for both research and diagnosis purposes. We analysed a total of 200 patients affected by ID and ID/ASD (84 with ID and 116 with ID and ASD) collected from January 2019 until the end of March 2021.

Genomic DNA from the parents was isolated from EDTA peripheral blood samples using MagCore HF16 (Diatech Lab Line, Jesi, Ancona, Italy) according to the manufacturer's instructions.

\subsection{Exome Sequencing}

Sample preparation was performed following the Illumina DNA Prep with Enrichment manufacturer protocol. A bead-based transposome complex is used to perform tagmentation, a process that fragments the genomic DNA and then tags it with adapter sequences in one step. After saturation with input DNA, the bead-based transposome complex fragments a set number of DNA molecules. This fragmentation provides flexibility to use a wide DNA input range to generate normalized libraries with a consistent tight fragment size distribution. Then a limited-cycle PCR adds adapter sequences to the ends of a DNA fragment. A subsequent target enrichment workflow is then applied. Following pooling, the double-stranded DNA libraries are denatured and biotinylated. Illumina Exome Panel v1.2 (CEX) probes are hybridized to the denatured library fragments. Then Streptavidin Magnetic Beads (SMB) capture the targeted library fragments within the regions of interest. Then the indexed libraries are eluted from beads and further amplified before sequencing. The exome sequencing analysis was performed on the Illumina NovaSeq6000 System (Illumina San Diego, CA, USA) according to the NovaSeq6000 System Guide. Reads were mapped against the hg19 reference genome using the Burrow-Wheeler aligner BWA [11]. Variant calling was obtained using an in-house pipeline which takes advantage of the GATK Best Practices workflow [12].

Prioritization of the variants was obtained excluding polymorphisms (minor allele frequency, MAF $<0.01$ ), synonymous variants, variants classified as benign or likely benign. Frameshift, stopgain, and splice site variants were prioritized as pathogenic. Missense variants were predicted to be damaging by CADD-Phred prediction tools. The potential impact of variants on splicing was evaluated using Alamut ${ }^{\circledR}$ Visual software-version 
2.11-0 (Interac-tive Biosoftware, Rouen, France), which employs five different algorithms: SpliceSiteFinder-like, MaxEntScan, NNSPLICE, GeneSplicer, and HumanSplicingFinder.

The following public databases were used for the interpretation of the variants: ClinVar (https://www.ncbi.nlm.nih.gov/clinvar/, accessed on 9 June 2021), LOVD (https:/ / databases.lovd.nl/shared/genes, accessed on 9 June 2021), the Human Genome Mutation Database (HGMD, http:/ / www.hgmd.cf.ac.uk/ac/index.php3, accessed on 9 June 2021).

\section{Results}

\subsection{Clinical Characteristics of Patients}

We enrolled 200 families (574 individuals total) with at least one proband with an unexplained diagnosis of ASD/ID-related phenotype. In particular, 181 families had one affected proband and 4 families had two affected probands. ES was sequenced to an average depth of $100 \times$, respectively, with $\sim 94 \%$ of bases covered $\geq 20 \times$. The study population had a mean age of 15 years and was $64 \%$ male $(128 / 200)$. We divided into four groups on the basis of the age $(0-10 ; 11-18 ; 19-30 ; 31-49)$ and gender (Figure 1$)$. All individuals displayed ID, 58\% (116/200) associated with ASD. The totality of the patients had been subjected to genetic testing prior to enrolment in this study. Our ID and ID/ASD cohort was characterized by the presence of additional associated clinical findings for $90 \%$ of the patients $(179 / 200)$. These included epilepsy $(n=41 / 200,20 \%)$, hypotonia $(n=10 / 200,5 \%)$, and MRI abnormalities observed in 13 patients. Craniofacial dysmorphisms $(n=106 / 200,53 \%)$ were found in $53 \%$ of the cases. The clinical descriptions of the 200 patients are summarized in Table S1.
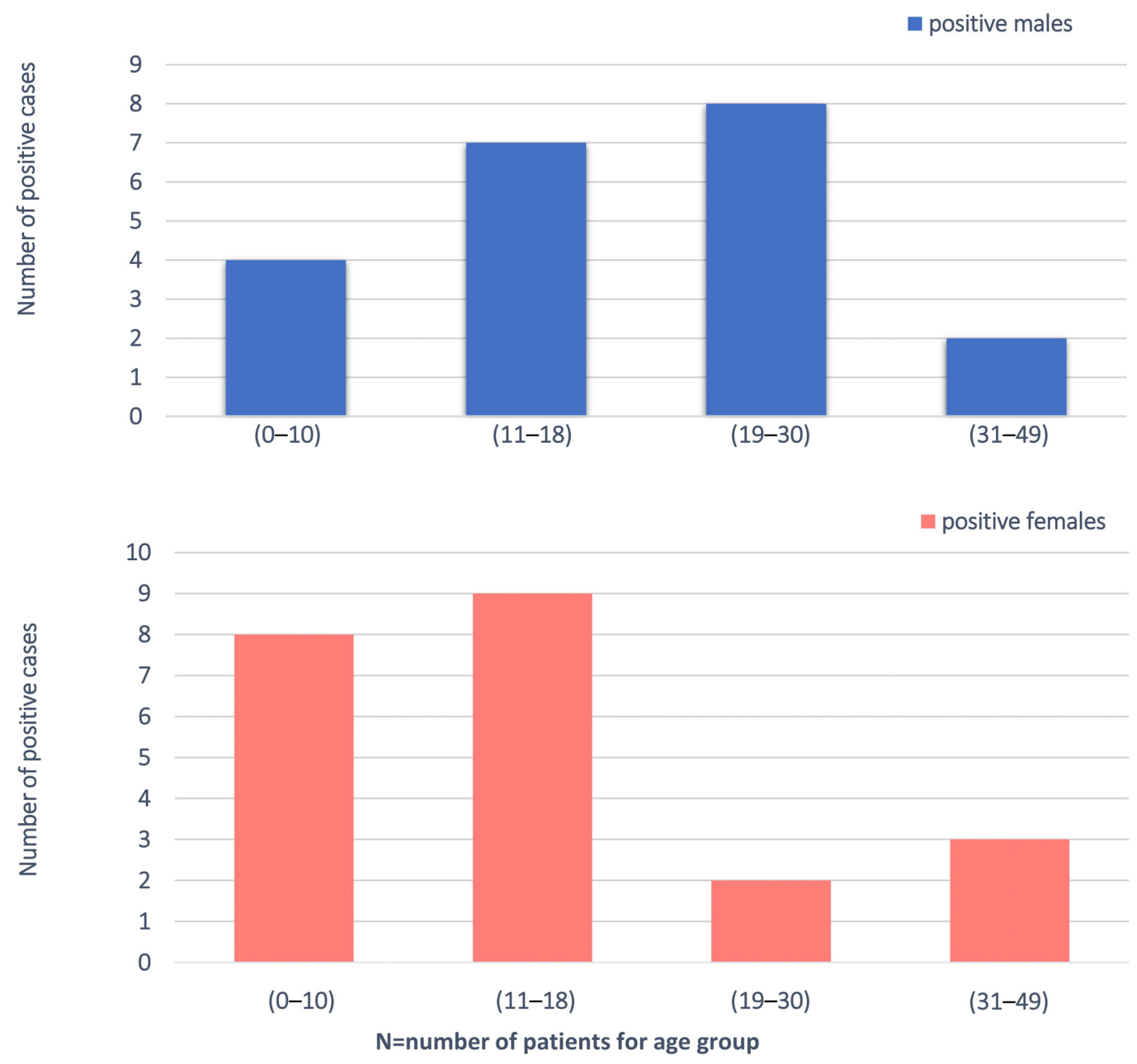

Figure 1. Demographic analysis of the cohort, filtered by age and gender. 


\section{2. $P / L P$ Variants Identified by ES}

ES was performed in 200 probands with ASD and/or ID and 41 different pathogenic/ likely pathogenic (P/LP) variants were identified with a detection rate of $22 \%$ (Table S2). Clinical features of patients with pathogenic variants in disease genes were described in Table 1.

Variants were classified based on frequency, mutation category, literature, and databases such as ClinVar. Affected individuals were categorized based on the number of parents that were sequenced along with the proband(s): proband-parent trios (184); duos with one parent (6); and proband-only singletons (10). Most P/LP variants were missense variants $(15 / 200 ; 8 \%)$, while $5 \%(9 / 200)$ were nonsense, $7 \%(13 / 200)$ frameshift and $2 \%$ $(4 / 200)$ splicing variants. Most (62\%) P/LP variations occurred de novo, while $29 \%$ of individuals inherited P/LP variants as heterozygotes or homozygotes. Four out of $200(2 \%)$ participants who harboured a P/LP result were sequenced with one or no biological parent and thus have unknown inheritance.

Mutations in the following genes were found in patients suffering from ID: ADNP, AP4M1, ATP1A3, BSCL2, CACNA1A, CSNK2B, CTU2, DEPDC5, DYRK1A, EFTUD2, HK1, IQSEC2, KANSL1, KIF1A, KMT2A, MBOAT7, MED13L, MMACHC, POGZ, PTPN11, RHOBTB2, SHANK3, SPG7, SPTBN2, TBCE, TUBA1A, WDR45, WFS1. In patients presenting autism and ID we found pathogenic variants in: BCOR, DDX3X, FGFR3, KCNQ3, SHANK3, SYNGAP1, TREX1, UPF3B, WFS1 (Table S2).

Two de novo P/LP variants were found in the new candidate genes: CACNA2D1 and GPR14 (Table S3), and the corresponding clinical pictures were reported in Table 2.

\subsection{Uncertain Variants Identified by ES}

We further reported 45 uncertain variants including in this number the variants that are currently considered to have an uncertain significance in the databases and other missense variants that have not been previously described in the scientific literature (Table S4). The effect on the encoded mutated proteins has been predicted using CADD (combined depletion annotation depletion). In our study the majority of uncertain and of P/LP variants fall in genes that play a role in the axon guidance and in the neurodevelopment processes (https:/ / reactome.org/PathwayBrowser/\#/, accessed on 6 July 2021). 
Table 1. Clinical features of patients with pathogenic variants in disease genes.

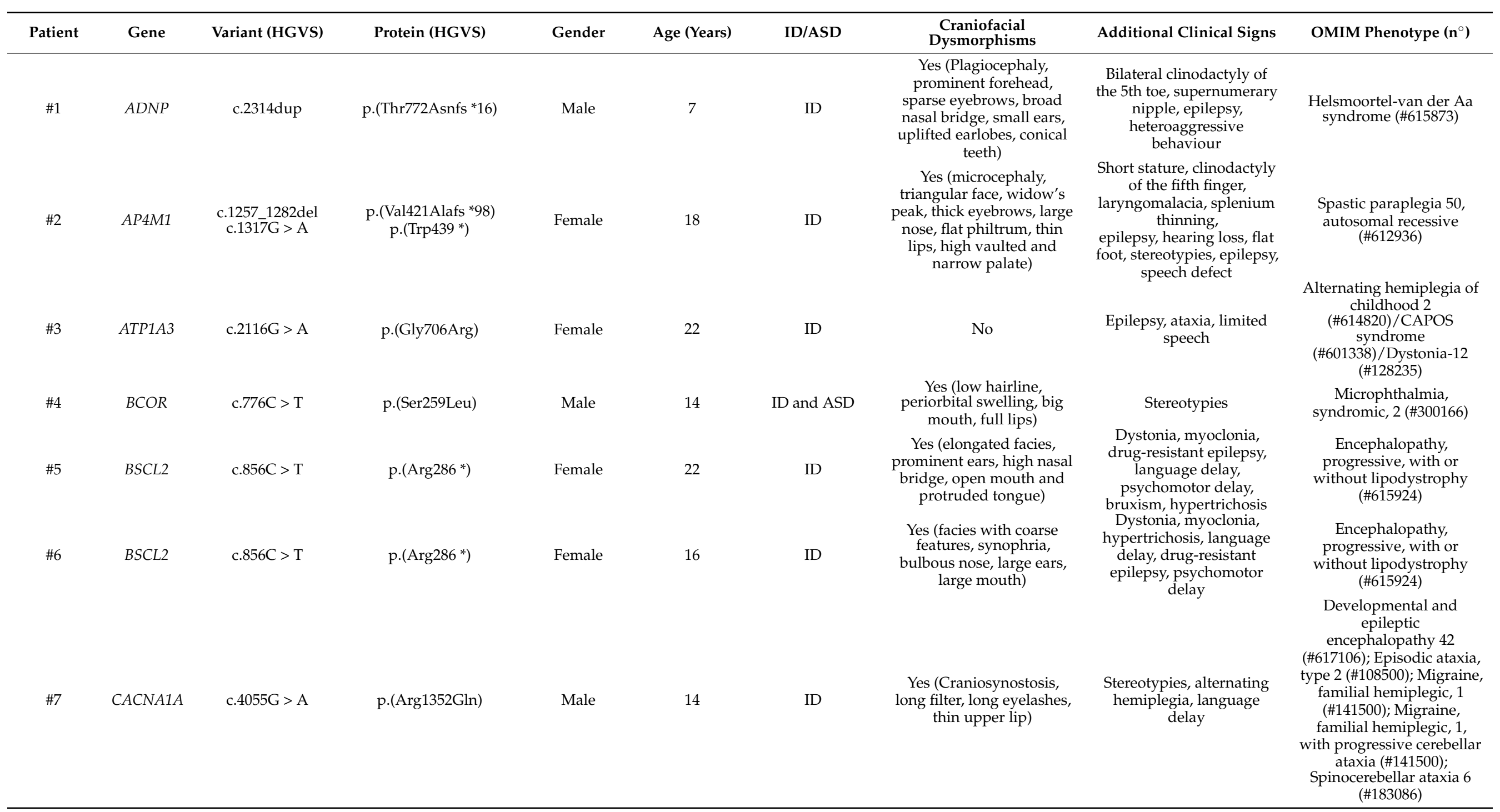


Table 1. Cont

\begin{tabular}{|c|c|c|c|c|c|c|c|c|c|}
\hline Patient & Gene & Variant (HGVS) & Protein (HGVS) & Gender & Age (Years) & ID/ASD & $\begin{array}{c}\text { Craniofacial } \\
\text { Dysmorphisms }\end{array}$ & Additional Clinical Signs & OMIM Phenotype $\left(\mathrm{n}^{\circ}\right)$ \\
\hline$\# 8$ & CSNK2B & c.170del & p.(Glu57Glyfs *15) & Female & 20 & ID & $\begin{array}{c}\text { Yes (Open mouth, } \\
\text { protruding tongue, narrow } \\
\text { palate, dental anomalies) }\end{array}$ & $\begin{array}{l}\text { Epilepsy, hypotonia, } \\
\text { developmental delay, } \\
\text { absent language }\end{array}$ & $\begin{array}{c}\text { Poirier-Bienvenu } \\
\text { neurodevelopmental } \\
\text { syndrome (\#618732) }\end{array}$ \\
\hline$\# 10$ & DEPDC5 & c. $1453 \mathrm{C}>\mathrm{T}$ & p. $\left(\operatorname{Arg} 485^{*}\right)$ & Male & 11 & ID & $\begin{array}{l}\text { Yes (low hairline, flattened } \\
\text { nasal bridge, bulbous } \\
\text { nasal tip, anteverted } \\
\text { nostrils, full lips, small and } \\
\text { widely spaced teeth, } \\
\text { anteverted ears) }\end{array}$ & Epilepsy, language delay & $\begin{array}{l}\text { Epilepsy, familial focal, } \\
\text { with variable foci } 1 \\
\text { (\#604364) }\end{array}$ \\
\hline$\# 11$ & $D D X 3 X$ & c. $976 \mathrm{C}>\mathrm{T}$ & p.(Arg326Cys) & Female & 12 & ID and ASD & $\begin{array}{l}\text { Yes (Microcephaly, long } \\
\text { face, smooth and long } \\
\text { philtrum, strabismus, up } \\
\text { slanting palpebral fissures) }\end{array}$ & $\begin{array}{l}\text { Developmental delay, } \\
\text { stereotypic hand } \\
\text { movements, hypotonia, } \\
\text { bruxism, sialorrhea, } \\
\text { corpus callosum } \\
\text { hypoplasia }\end{array}$ & $\begin{array}{l}\text { Intellectual developmental } \\
\text { disorder, X-linked, } \\
\text { syndrome, Snijders Blok } \\
\text { type (\#300958) }\end{array}$ \\
\hline$\# 12$ & DYRK1A & c. $1669 \mathrm{C}>\mathrm{T}$ & p. $\left(\mathrm{G} \ln 557^{*}\right)$ & Male & 16 & ID & $\begin{array}{l}\text { Yes (Microcephaly, narrow } \\
\text { forehead, frontal bossing, } \\
\text { depressed nasal bridge, } \\
\text { short philtrum, } \\
\text { prognathism) }\end{array}$ & $\begin{array}{c}\text { Hypotonia, epilepsy, } \\
\text { stereotypies, absent } \\
\text { language }\end{array}$ & $\begin{array}{l}\text { Mental retardation, } \\
\text { autosomal dominant } 7 \\
(\# 614104)\end{array}$ \\
\hline$\# 13$ & EFTUD2 & c. $702+1 G>A$ & NA & Female & 22 & ID & $\begin{array}{l}\text { Yes (prominent columella, } \\
\text { sloping forehead, high and } \\
\text { scattered eyebrows, deeply } \\
\text { set eyes, nose with } \\
\text { anteverted nostrils, narrow } \\
\text { palate, prominent incisors, } \\
\text { prominent ears) }\end{array}$ & $\begin{array}{l}\text { Hypertrichosis, small } \\
\text { hands and feet, joint } \\
\text { hyperextensivity }\end{array}$ & $\begin{array}{c}\text { Mandibulofacial } \\
\text { dysostosis, Guion-Almeida } \\
\text { type (\#603892) }\end{array}$ \\
\hline$\# 14$ & FGFR3 & c. $749 \mathrm{C}>\mathrm{G}$ & p.(Pro250Arg) & Female & 4 & ID and ASD & $\begin{array}{c}\text { Yes (craniosynostosis, flat } \\
\text { forehead, down slanting } \\
\text { palpebral fissures, high } \\
\text { nasal bridge) }\end{array}$ & $\begin{array}{l}\text { Language delay, } \\
\text { hyperchromic and } \\
\text { hypochromic spots }\end{array}$ & $\begin{array}{l}\text { Muenke syndrome } \\
\text { (\#602849) CATSHL } \\
\text { syndrome (\#610474) }\end{array}$ \\
\hline
\end{tabular}


Table 1. Cont

\begin{tabular}{|c|c|c|c|c|c|c|c|c|c|}
\hline Patient & Gene & Variant (HGVS) & Protein (HGVS) & Gender & Age (Years) & ID/ASD & $\begin{array}{c}\text { Craniofacial } \\
\text { Dysmorphisms }\end{array}$ & Additional Clinical Signs & OMIM Phenotype $\left(\mathrm{n}^{\circ}\right)$ \\
\hline \#15 & HK1 & c. $1367 \mathrm{C}>\mathrm{T}$ & p.(Thr456Met) & Male & 16 & ID & $\begin{array}{l}\text { Yes (proptosis, long } \\
\text { eyelashes, synophria, } \\
\text { narrow palate, dental } \\
\text { anomalies, small mouth, } \\
\text { and full lips) }\end{array}$ & $\begin{array}{l}\text { Bilateral cryptorchidism, } \\
\text { psychomotor delay, poor } \\
\text { vision, ventricular system } \\
\text { dilation, immature } \\
\text { hippocampal } \\
\text { structures, spastic } \\
\text { paraparesis, epilepsy, } \\
\text { hypothonia, absent } \\
\text { language }\end{array}$ & $\begin{array}{l}\text { Neurodevelopmental } \\
\text { disorder with } \\
\text { visual defects and brain } \\
\text { anomalies } \\
\text { (\#618547) }\end{array}$ \\
\hline$\# 16$ & IQSEC2 & c.3780del & p. (Gln1261Serfs *136) & Female & 22 & ID & No & $\begin{array}{l}\text { Strabismus, cerebral } \\
\text { atrophy, sialorrhea, motor } \\
\text { problems, bruxism }\end{array}$ & $\begin{array}{l}\text { Mental retardation, } \\
\text { X-linked 1/78 (\#309530) }\end{array}$ \\
\hline$\# 17$ & KANSL1 & c.985_986del & p.(Leu329Glufs *22) & Female & 6 & ID & $\begin{array}{l}\text { Yes (cleft palate, deeply set } \\
\text { eyes, high nasal bridge, } \\
\text { bulbous nasal tip) }\end{array}$ & $\begin{array}{l}\text { Language delay, } \\
\text { epilepsy, aggressiveness }\end{array}$ & $\begin{array}{l}\text { Koolen-De Vries syndrome } \\
\quad(\# 610443)\end{array}$ \\
\hline$\# 18$ & KCNQ3 & c. $688 \mathrm{C}>\mathrm{T}$ & p.(Arg230Cys) & Male & 39 & ID and ASD & $\begin{array}{l}\text { Yes (triangular facies, high } \\
\text { nasal bridge, bulbous } \\
\text { nasal tip, open mouth, } \\
\text { micrognathia, narrow } \\
\text { and downturned eyelids) }\end{array}$ & $\begin{array}{c}\text { Stereotypies, } \\
\text { aggressiveness, bladder } \\
\text { anomalies }\end{array}$ & $\begin{array}{c}\text { Seizures, benign neonatal, } \\
2(\# 121201)\end{array}$ \\
\hline \#19 & KIF1A & c. $37 \mathrm{C}>\mathrm{T}$ & p.(Arg13Cys) & Female & 16 & ID & 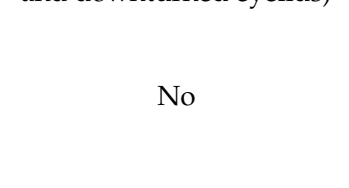 & $\begin{array}{c}\text { Spastic paraparesis, } \\
\text { behaviour disorder, slight } \\
\text { enlargement of the } \\
\text { interfolial } \\
\text { spaces of the cerebellar } \\
\text { hemispheres, hypertone }\end{array}$ & $\begin{array}{c}\text { Spastic paraplegia 30, } \\
\text { autosomal dominant } \\
(\# 610357)\end{array}$ \\
\hline \#20 & KIF1A & c. $914 \mathrm{C}>\mathrm{T}$ & p.(Pro305Leu) & Female & 10 & ID & $\begin{array}{l}\text { Yes (sparse eyebrows, } \\
\text { small nose, thin upper lip, } \\
\text { dental anomalies, chubby } \\
\text { cheeks) }\end{array}$ & $\begin{array}{l}\text { Language delay, cerebellar } \\
\text { and worm atrophy, } \\
\text { psychomotor delay, brain } \\
\text { abnormalities, } \\
\text { hypertrichosis, bilateral } \\
\text { clinodactyly of the } \\
\text { second/third/fourth/fifth } \\
\text { toes }\end{array}$ & $\begin{array}{c}\text { Spastic paraplegia 30, } \\
\text { autosomal dominant } \\
(\# 610357)\end{array}$ \\
\hline \#21 & KIF1A & c. $914 \mathrm{C}>\mathrm{T}$ & p.(Pro305Leu) & Male & 49 & ID & No & $\begin{array}{c}\text { Ataxia, spastic paraparesis, } \\
\text { angioma, nystagmus, } \\
\text { epilepsy }\end{array}$ & $\begin{array}{c}\text { Spastic paraplegia 30, } \\
\text { autosomal dominant } \\
(\# 610357)\end{array}$ \\
\hline$\# 22$ & KMT2A & c.5256delA & p.(Ala1753Profs*70) & Male & 16 & ID & $\begin{array}{l}\text { Yes (thick eyebrows, } \\
\text { narrow and short eyelids, } \\
\text { depressed helix, nose with } \\
\text { bulbous tip, thick lips) }\end{array}$ & $\begin{array}{l}\text { Hypertrichosis of the legs, } \\
\text { arms and lumbar region, } \\
\text { large hands, short fifth ray } \\
\text { of the foot }\end{array}$ & $\begin{array}{l}\text { Wiedemann-Steiner } \\
\text { syndrome (\#605130) }\end{array}$ \\
\hline
\end{tabular}


Table 1. Cont

\begin{tabular}{|c|c|c|c|c|c|c|c|c|c|}
\hline Patient & Gene & Variant (HGVS) & Protein (HGVS) & Gender & Age (Years) & ID/ASD & $\begin{array}{c}\text { Craniofacial } \\
\text { Dysmorphisms }\end{array}$ & Additional Clinical Signs & OMIM Phenotype $\left(\mathrm{n}^{\circ}\right)$ \\
\hline$\# 23$ & MBOAT7 & c. $477 \mathrm{C}>\mathrm{G}$ & p. $($ Tyr159*) & Female & 2 & ID & No & $\begin{array}{l}\text { Motor stereotypies, } \\
\text { epilepsy, thinning of the } \\
\text { corpus callosum, } \\
\text { ventricular enlargement }\end{array}$ & $\begin{array}{c}\text { Mental retardation, } \\
\text { autosomal recessive } 57 \\
\text { (\#617188) }\end{array}$ \\
\hline$\# 24$ & $M E D 13 L$ & $c .72+1 G>T$ & NA & Female & 49 & ID & $\begin{array}{l}\text { Yes (sparse eyebrows, } \\
\text { hypertelorism, narrow } \\
\text { eyelids, gingival } \\
\text { hypertrophy) }\end{array}$ & $\begin{array}{l}\text { Syndactyly of the second } \\
\text { and third toes, small feet, } \\
\text { hypertrophy of the limbs } \\
\text { and truncal obesity, } \\
\text { strabismus }\end{array}$ & $\begin{array}{c}\text { Mental retardation and } \\
\text { distinctive facial features } \\
\text { with or without cardiac } \\
\text { defects (\#616789) }\end{array}$ \\
\hline \#25 & $M M A C H C$ & c. $440 \mathrm{G}>\mathrm{C}$ & p.(Gly147Ala) & Male & 12 & ID & $\begin{array}{l}\text { Yes (synophria, horizontal } \\
\text { eyebrows, wide nasal tip, } \\
\text { anteverted nostrils, long } \\
\text { filter, dental anomalies) }\end{array}$ & $\begin{array}{l}\text { Spastic paraparesis, } \\
\text { language delay, } \\
\text { polyneuropathy }\end{array}$ & $\begin{array}{l}\text { Methylmalonic aciduria } \\
\text { and homocystinuria, cblC } \\
\text { type (\#277400) }\end{array}$ \\
\hline \#26 & POGZ & c.1180_1181del & p.(Met394Valfs *9) & Female & 6 & ID & $\begin{array}{l}\text { Yes (microcephaly, deeply } \\
\text { set eyes, nose with } \\
\text { bulbous tip, anteverted } \\
\text { nostrils, full lips) }\end{array}$ & $\begin{array}{c}\text { Hyperactivity, } \\
\text { blepharophimosis, } \\
\text { brachydactyly, nail } \\
\text { hypoplasia, } \\
\text { kidney abnormalities, } \\
\text { language delay }\end{array}$ & $\begin{array}{l}\text { White-Sutton syndrome } \\
\qquad(\# 616364)\end{array}$ \\
\hline \#27 & POGZ & c.1180_1181del & p.(Met394Valfs *9) & Male & 12 & ID & $\begin{array}{l}\text { Yes (narrow bitemporal } \\
\text { diameter, narrow and } \\
\text { upward eyelid rims, deep } \\
\text { philtrum, progatism, } \\
\text { exaggerated Cupid's bow, } \\
\text { buccal rim pointing } \\
\text { downwards, uplifted ear } \\
\text { lobe) }\end{array}$ & Hypotonia, obesity & $\begin{array}{l}\text { White-Sutton syndrome } \\
\qquad(\# 616364)\end{array}$ \\
\hline \#28 & POGZ & c.1180_1181del & p. (Met394Valfs *9) & Female & 44 & ID & No & $\begin{array}{c}\text { Microcephaly, } \\
\text { brachydactyly, nail } \\
\text { hypoplasia }\end{array}$ & $\begin{array}{l}\text { White-Sutton syndrome } \\
\qquad(\# 616364)\end{array}$ \\
\hline \#29 & PTPN11 & c. $1471 \mathrm{C}>\mathrm{A}$ & p.(Pro491Thr) & Male & 2 & ID & $\begin{array}{c}\text { Yes (high forehead, low-set } \\
\text { ears with large, } \\
\text { downward-pointing } \\
\text { auricles, } \\
\text { down-slanting eyelids, } \\
\text { broad nasal tip, long and } \\
\text { thick filter, exaggerated } \\
\text { Cupid's bow) }\end{array}$ & $\begin{array}{l}\text { Café au lait spots in the } \\
\text { thoracic and lumbar } \\
\text { region, nail hypoplasia of } \\
\text { the fifth toe, } \\
\text { neurodevelopmental delay, } \\
\text { cryptorchidism }\end{array}$ & $\begin{array}{l}\text { Noonan syndrome } 1 \\
\quad(\# 163950)\end{array}$ \\
\hline
\end{tabular}


Table 1. Cont

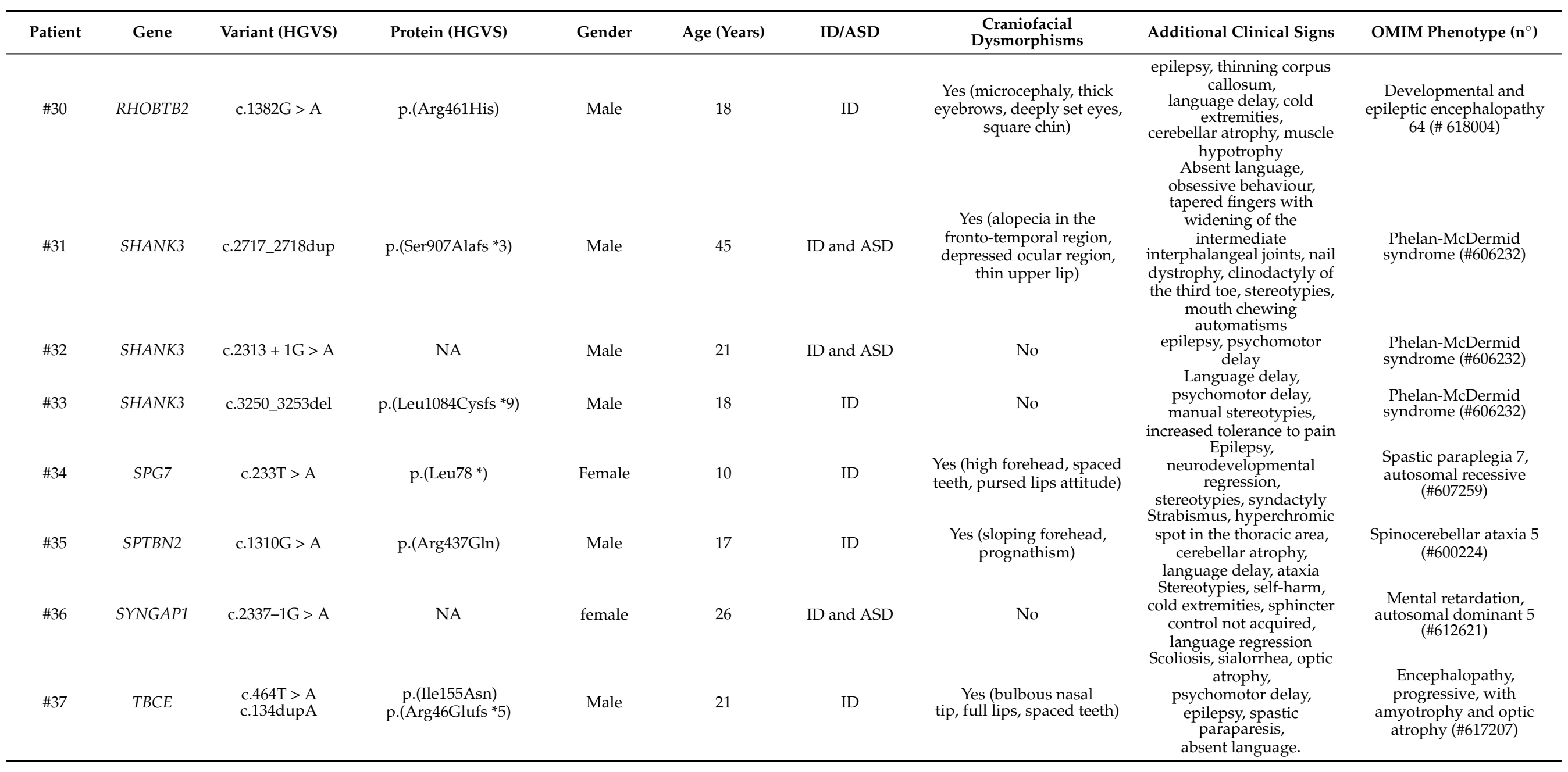


Table 1. Cont.

\begin{tabular}{|c|c|c|c|c|c|c|c|c|c|}
\hline Patient & Gene & Variant (HGVS) & Protein (HGVS) & Gender & Age (Years) & ID/ASD & $\begin{array}{c}\text { Craniofacial } \\
\text { Dysmorphisms }\end{array}$ & Additional Clinical Signs & OMIM Phenotype $\left(n^{\circ}\right)$ \\
\hline \#38 & TREX1 & c.558_573del & p. $($ Phe186Leufs *24) & Male & 10 & ID and ASD & No & $\begin{array}{l}\text { Gastroesophageal reflux, } \\
\text { sphincter control not } \\
\text { acquired, stereotyped } \\
\text { behaviour }\end{array}$ & $\begin{array}{l}\text { Aicardi-Goutieres } \\
\text { syndrome } 1 \text { dominant and } \\
\text { recessive (\#225750) }\end{array}$ \\
\hline \#39 & TUBA1A & c. $352 \mathrm{G}>\mathrm{A}$ & p.(Val118Met) & Male & 9 & ID & $\begin{array}{l}\text { Yes (advanced hairline, } \\
\text { long and thick eyebrows, } \\
\text { anteverted nostrils, long } \\
\text { filter, thin upper lip) }\end{array}$ & $\begin{array}{l}\text { Hypoplasia of the } \\
\text { cerebellar vermis, thinned } \\
\text { corpus callosum, } \\
\text { cerebellar asymmetry, } \\
\text { angioma, hypertrichosis, } \\
\text { epilepsy, apraxia, ataxia, } \\
\text { and psychomotor delay }\end{array}$ & Lissencephaly 3 (\#611603) \\
\hline$\# 40$ & UРF3В & c. $1288 \mathrm{C}>\mathrm{T}$ & p. $(\operatorname{Arg} 430 *)$ & Male & 7 & ID and ASD & $\begin{array}{l}\text { Yes (Wide forehead, } \\
\text { arched eyebrows, deeply } \\
\text { set eyes, pointed chin) }\end{array}$ & $\begin{array}{l}\text { Limited speech, } \\
\text { neurodevelopmental delay }\end{array}$ & $\begin{array}{c}\text { Mental retardation, } \\
\text { X-linked syndromic } 14 \\
(\# 300676)\end{array}$ \\
\hline \#41 & WDR45 & c.66del & p.(Cys23Alafs *15) & Female & 20 & ID & $\begin{array}{c}\text { Yes (thick eyebrows, } \\
\text { prominent upper arch, } \\
\text { hyperemic gums, high } \\
\text { palate) }\end{array}$ & $\begin{array}{l}\text { Limited speech, scoliosis, } \\
\text { locomotor impairment, } \\
\text { manual stereotypies, } \\
\text { tapered fingers }\end{array}$ & $\begin{array}{c}\text { Neurodegeneration with } \\
\text { brain } \\
\text { iron accumulation } 5 \\
(\# 300894)\end{array}$ \\
\hline \#42 & WFS1 & c. $124 \mathrm{C}>\mathrm{T}$ & p. $(\operatorname{Arg} 42 *)$ & Male & 6 & ID and ASD & No & $\begin{array}{l}\text { Language delay, } \\
\text { oppositional and } \\
\text { provocative behaviour }\end{array}$ & $\begin{array}{l}\text { Wolfram-like syndrome, } \\
\text { autosomal dominant } \\
\qquad \# 614296)\end{array}$ \\
\hline \#43 & WFS1 & c.1230_1233del & p.(Leu412Serfs *29) & Female & 14 & ID & $\begin{array}{l}\text { Yes (microcephaly, } \\
\text { synophria, long eyelashes, } \\
\text { bulbous nasal tip) }\end{array}$ & $\begin{array}{c}\text { Hypertrichosis, } \\
\text { drug-resistant seizures, } \\
\text { spastic tetraparesis, renal } \\
\text { failure. }\end{array}$ & $\begin{array}{l}\text { Wolfram-like syndrome, } \\
\text { autosomal dominant } \\
(\# 614296)\end{array}$ \\
\hline
\end{tabular}

* means change in a stop codon. 
Table 2. Clinical features of patients with pathogenic variants in the new candidate genes.

\begin{tabular}{|c|c|c|c|c|c|c|c|c|}
\hline Patient & Gene & Variant (HGVS) & Protein (HGVS) & Gender & Age (Years) & ID/ASD & $\begin{array}{c}\text { Craniofacial } \\
\text { Dysmorphisms }\end{array}$ & $\begin{array}{c}\text { Additional } \\
\text { Clinical Signs }\end{array}$ \\
\hline$\# 44$ & CACNA2D1 & c.659-2_659-1insT & NA & Female & 14 & ID & $\begin{array}{c}\text { Yes (deeply set eyes, } \\
\text { squared facies, } \\
\text { bulbous nasal tip, full } \\
\text { lips, horizontal } \\
\text { eyebrows, enlarged } \\
\text { nasal bridge, mouth } \\
\text { with downward } \\
\text { corners, anteverted } \\
\text { nostrils) }\end{array}$ & $\begin{array}{c}\text { Language delay, } \\
\text { epilepsy }\end{array}$ \\
\hline$\# 45$ & $\begin{array}{l}\text { GPR14 (before } \\
\text { UTS2R) }\end{array}$ & c. $844 \mathrm{C}>\mathrm{T}$ & p. $\left(\mathrm{G} \ln 282^{*}\right)$ & Male & 6 & ID and ASD & $\begin{array}{l}\text { Yes (long and large } \\
\text { nose, broad nasal } \\
\text { bridge, anteverted } \\
\text { nostrils, deep } \\
\text { philtrum) }\end{array}$ & $\begin{array}{l}\text { Frequent } \\
\text { infections }\end{array}$ \\
\hline
\end{tabular}




\section{Discussion}

This study emphasizes the clinical diagnostic relevance of ES in patients with ID and/or autism with additional clinical features. In particular, in a cohort of 200 patients, we reached a diagnostic yield of $22 \%(43 / 200)$ with a higher rate in ID patients $(33 / 84 ; 39 \%)$ with respect to ID and ASD patients (10/116; $9 \%)$. The diagnostic yield is lower with respect to other studies that employ ES in neurodevelopmental disorder (30-43\%) [13]. ES has technological limitations, including the inability to detect noncoding variants, copy number variants (CNVs), epigenetic changes, and trinucleotide repeat expansion [14]. In our cohort, $30 \%$ of cases have not been screened for $\mathrm{CNVs}$ and this could have underestimated the presence of other pathogenic genetic alterations, in particular in patients with ASD. Until recently, whole genome chromosomal microarray was recommended as a first-tier clinical genetic test for detecting disease-causing CNVs in individuals with ASD [15-17].

Most of the previous diagnoses failed for the atypical phenotypic presentation of well-established genetic syndromes. The DDX3X mutated patient (\#11) shows Rett-like spectrum features with typical hand-washing stereotypes and was initially screened for mutations in MECP2, FOXG1 and CDKL5 genes [18-21]. Differently, a hundred patients are reported in literature mutated in DDX3X with various clinical features including hypotonia, movement disorder, behavioural problems, corpus callosum hypoplasia and epilepsy $[22,23]$. Another atypical clinical picture was manifested by a patient bearing the mutation c.688C > T (p.(Arg230Cys)) in KCNQ3, who did not suffer from any status epilepticus but showing ID, autism, stereotypies, aggressiveness, bladder anomalies; he also presented craniofacial dysmorphisms (patient \#18). KCNQ3 pathogenic alterations are generally linked to the occurrence of seizures but recently patients with no EEG abnormalities have been described [24]. KIF1A gene was found altered in three unrelated cases with different phenotypic presentations (patients \#19, \#20, \#21). KIF1A mutations cause NESCAV syndrome (NESCAVS), a neurodegenerative disorder characterized by global developmental delay, progressive spasticity, ID, speech delay, learning disabilities and/or behavioural abnormalities [25]. The mutation c.37C > T (p.(Arg13Cys)) in KIF1A was found in patient \#19 with spastic paraparesis, behaviour disorder, slight enlargement of the interfolial spaces of the cerebellar hemispheres, hypertone and no craniofacial dysmorphisms. In particular the same mutation c.914C > T (p.(Pro305Leu)) was shown in two different patients (\#20, \#21). One of these, presented language delay, cerebellar and vermis atrophy, psychomotor delay, brain abnormalities, hypertrichosis, bilateral clinodactyly, and facial dysmorphisms (patient \#20). The other showed ataxia, spastic paraparesis, angioma, nystagmus, and seizures (patient \#21). Mutations in POGZ are associated with the White-Sutton syndrome, which is a neurodevelopmental disorder characterized by delayed psychomotor development and a characteristic constellation of dysmorphic facial features [26]. Additional features may include hypotonia, sensorineural hearing impairment, visual defects, joint laxity, and gastrointestinal difficulties [27]. The pathogenic variant c.1180_1181del (p.(Met394Valfs*9)) was carried by two siblings and their mother (\#26, \#27, \#28). The sister exhibited craniofacial dysmorphisms and ID; she also showed hyperactivity, blepharophimosis, brachydactyly, nail hypoplasia, kidney abnormalities and language delay as additional clinical signs (patient \#26). The brother was affected by ID, hypotonia, obesity and had some craniofacial dysmorphisms (patient \#27). Their mother instead displayed ID, microcephaly, brachydactyly, and nail hypoplasia (patient \#28). Another example, SHANK3 was found altered in three unrelated patients (\#31, \#32, \#33). Mutations in SHANK3 cause Phelan-McDermid syndrome, a developmental disorder with variable features including neonatal hypotonia, global developmental delay, absent to severely delayed speech, autistic behaviour, and minor dysmorphic features [28-30]. One of the three mutated patients did not show neither autism nor dysmorphic features and was initially classified as a "non-syndromic" ID case.

The following new candidate genes for ID/ASD have emerged: CACNA2D1 and GPR14. They all show de novo truncating variants (patients \#44-\#45). CACNA2D1 encodes the alpha-2/delta subunit of skeletal muscle and brain voltage-dependent calcium 
channels [31]. A genomic aberration affecting the CACNA2D1 gene has been previously characterized in patients with epilepsy and ID, pinpointing the gene as an interesting candidate gene for these clinical features [32]. Mice-bearing point mutations in the CACNA2D1 gene have an abnormal central nervous system synaptic transmission [33]. GPR14 gene, encoding the orphan G protein-coupled receptor 14 for Urotensin II, is widely expressed in the brain and spinal cord [34]. We found 45 variants of unknown significance (VUS) in 40 patients. These variants are $22 / 84(26 \%)$ in ID patients and 18/116 $(16 \%)$ in ID/ASD patients. These variants are mostly missense $42 / 45$ (93\%) and CADD $\geq 25$ in 20/42 (48\%) of cases. With increased knowledge over time, exome reanalysis may change the clinical interpretation of a VUS. Thus, it is important to list all the VUS, analyse them periodically and write a report in case of changes to provide a timely response for patients and families. An accurate molecular diagnosis allows for precise genetic counselling and has the potential to change clinical management.

\section{Conclusion}

ES was able to avoid a sort of "diagnostic odyssey" for a significant fraction of families consisting in the step-by-step application of the traditional genetic methods. ES revealed atypical phenotypic presentations and new candidate genes for ID/ASD. Further studies are needed to better characterize the contribution of new candidates and to show how their haploinsufficiency can determine ID/ASD.

Supplementary Materials: The following are available online at https://www.mdpi.com/article/10 .3390/brainsci11070936/s1, Table S1: Clinical features of the 200 ID and ID/ASD patients; Table S2: $\mathrm{P} / \mathrm{LP}$ variants identified in disease genes; Table S3: P/LP variants identified in candidate disease genes; Table S4: Uncertain variants identified by ES

Author Contributions: F.V., L.P.B., G.D., A.G., A.R. and F.A. have made substantial contributions to conceptions and design and have been involved in drafting the paper. R.T., S.R., C.F., M.B. (Mirella Bruttini), E.B., K.Z., S.F. has made substantial contributions to acquisition and analysis of the data. M.A.M., F.M., A.M.P., F.F., M.B. (Margherita Baldassarri), C.L.R., A.F., V.L., made a clinical evaluation. All authors have given final approval of the version to be published and agree to be accountable for all aspects of the work in ensuring that questions related to the accuracy or integrity of any part of the work are appropriately investigated and resolved.

Funding: This research received no external fundings.

Institutional Review Board Statement: Ethical review and approval were not required for the study on human participants in accordance with local legislation and institutional requirements.

Informed Consent Statement: The patients/participants provided their written informed consent to participate in this study.

Data Availability Statement: NGS data has been deposited in publicly accessible repositories. The data can be found here: http://nigdb.cineca.it/.

Acknowledgments: We are grateful to our patient for the cooperation. The "Cell lines and DNA bank of Rett Syndrome, X-linked mental retardation, and other genetic diseases," member of the Telethon Network of Genetic Biobanks (project nos. GTB12001 and GFB18001), funded by Telethon Italy, and of the EuroBioBank network provided us with specimens.

Conflicts of Interest: Two of several authors of this publication are members of the European Reference Network for rare malformation syndromes and rare intellectual and neurodevelopmental disorders, ERN-ITHACA.

\section{References}

1. Tassé, M.J.; Luckasson, R.; Nygren, M. AAIDD proposed recommendations for ICD-11 and the condition previously known as mental retardation. Intellect. Dev. Disabil. 2013, 51, 127-131. [CrossRef] [PubMed]

2. Kolset, S.O. Intellectual disability and nutrition-related health. EMBO Mol. Med. 2020, 12. [CrossRef]

3. Maulik, P.K.; Mascarenhas, M.N.; Mathers, C.D.; Dua, T.; Saxena, S. Prevalence of intellectual disability: A meta-analysis of population-based studies. Res. Dev. Disabil. 2011, 32, 419-436. [CrossRef] 
4. Chiurazzi, P.; Kiani, A.K.; Miertus, J.; Barati, S.; Manara, E.; Paolacci, S.; Stuppia, L.; Gurrieri, F.; Bertelli, M. Genetic analysis of intellectual disability and autism. Acta Biomed. 2020, 91, e2020003. [CrossRef] [PubMed]

5. Sharma, S.R.; Gonda, X.; Tarazi, F.I. Autism Spectrum Disorder: Classification, diagnosis and therapy. Pharmacol. Ther. 2018, 190, 91-104. [CrossRef] [PubMed]

6. Baio, J.; Wiggins, L.; Christensen, D.L.; Maenner, M.J.; Daniels, J.; Warren, Z.; Kurzius-Spencer, M.; Zahorodny, W.; Rosenberg, C.R.; White, T.; et al. Prevalence of autism spectrum disorder among children aged 8 Years-Autism and developmental disabilities monitoring network, 11 Sites, United States, 2014. MMWR Surveill. Summ. 2018, 67, 1-23. [CrossRef]

7. Zablotsky, B.; Black, L.I.; Maenner, M.J.; Schieve, L.A.; Danielson, M.L.; Bitsko, R.H.; Blumberg, S.J.; Kogan, M.D.; Boyle, C.A. Prevalence and trends of developmental disabilities among children in the United States: 2009-2017. Pediatrics 2019, 144, e20190811. [CrossRef] [PubMed]

8. Baranova, J.; Dragunas, G.; Botellho, M.C.S.; Ayub, A.L.P.; Bueno-Alves, R.; Alencar, R.R.; Papaiz, D.D.; Sogayar, M.C.; Ulrich, H.; Correa, R.G. Autism Spectrum Disorder: Signaling Pathways and Prospective Therapeutic Targets; Springer: Berlin/Heidelberg, Germany, 2021; Volume 41, ISBN 0123456789.

9. Thevenon, J.; Duffourd, Y.; Masurel-Paulet, A.; Lefebvre, M.; Feillet, F.; El Chehadeh-Djebbar, S.; St-Onge, J.; Steinmetz, A.; Huet, F.; Chouchane, M.; et al. Diagnostic odyssey in severe neurodevelopmental disorders: Toward clinical whole-exome sequencing as a first-line diagnostic test. Clin. Genet. 2016, 89, 700-707. [CrossRef] [PubMed]

10. Munnich, A.; Demily, C.; Frugère, L.; Duwime, C.; Malan, V.; Barcia, G.; Vidal, C.; Throo, E.; Besmond, C.; Hubert, L.; et al. Impact of on-site clinical genetics consultations on diagnostic rate in children and young adults with autism spectrum disorder. Mol. Autism 2019, 10, 1-10. [CrossRef]

11. Li, H.; Durbin, R. Fast and accurate long-read alignment with Burrows-Wheeler transform. Bioinformatics 2010, 26, 589-595. [CrossRef] [PubMed]

12. Poplin, R.; Ruano-Rubio, V.; DePristo, M.; Fennell, T.; Carneiro, M.; Van der Auwera, G.; Kling, D.; Gauthier, L.; Levy-Moonshine, A.; Roazen, D.; et al. Scaling accurate genetic variant discovery to tens of thousands of samples. bioRxiv 2017. [CrossRef]

13. Srivastava, S.; Love-Nichols, J.A.; Dies, K.A.; Ledbetter, D.H.; Martin, C.L.; Chung, W.K.; Firth, H.V.; Frazier, T.; Hansen, R.L.; Prock, L.; et al. Meta-analysis and multidisciplinary consensus statement: Exome sequencing is a first-tier clinical diagnostic test for individuals with neurodevelopmental disorders. Genet. Med. 2019, 21, 2413-2421. [CrossRef] [PubMed]

14. Nolan, D.; Carlson, M. Whole exome sequencing in pediatric neurology patients: Clinical implications and estimated cost analysis. J. Child Neurol. 2016, 31, 887-894. [CrossRef]

15. Miller, D.T.; Adam, M.P.; Aradhya, S.; Biesecker, L.G.; Brothman, A.R.; Carter, N.P.; Church, D.M.; Crolla, J.A.; Eichler, E.E.; Epstein, C.J.; et al. Consensus statement: Chromosomal microarray is a first-tier clinical diagnostic test for individuals with developmental disabilities or congenital anomalies. Am. J. Hum. Genet. 2010, 86, 749-764. [CrossRef] [PubMed]

16. Battaglia, A.; Doccini, V.; Bernardini, L.; Novelli, A.; Loddo, S.; Capalbo, A.; Filippi, T.; Carey, J.C. Confirmation of chromosomal microarray as a first-tier clinical diagnostic test for individuals with developmental delay, intellectual disability, autism spectrum disorders and dysmorphic features. Eur. J. Paediatr. Neurol. 2013, 17, 589-599. [CrossRef] [PubMed]

17. Volkmar, F.R.; McPartland, J.C. From Kanner to DSM-5: Autism as an evolving diagnostic concept. Annu. Rev. Clin. Psychol. 2014, 10, 193-212. [CrossRef] [PubMed]

18. Ariani, F.; Hayek, G.; Rondinella, D.; Artuso, R.; Mencarelli, M.A.; Spanhol-Rosseto, A.; Pollazzon, M.; Buoni, S.; Spiga, O.; Ricciardi, S.; et al. FOXG1 is responsible for the congenital variant of Rett syndrome. Am. J. Hum. Genet. 2008, 83, 89-93. [CrossRef]

19. Renieri, A.; Meloni, I.; Longo, I.; Ariani, F.; Mari, F.; Pescucci, C.; Cambi, F. Rett syndrome: The complex nature of a monogenic disease. J. Mol. Med. 2003, 81, 346-354. [CrossRef] [PubMed]

20. Amir, R.E.; Van Den Veyver, I.B.; Wan, M.; Tran, C.Q.; Francke, U. Rett syndrome is caused by mutations in X-linked. Nat. Genet. 1999, 23, 185-188. [CrossRef]

21. Scala, E.; Ariani, F.; Mari, F.; Caselli, R.; Pescucci, C.; Longo, I.; Meloni, I.; Giachino, D.; Bruttini, M.; Hayek, G.; et al. CDKL5/STK9 is mutated in Rett syndrome variant with infantile spasms. J. Med. Genet. 2005, 2, 103-107. [CrossRef] [PubMed]

22. Snijders Blok, L.; Madsen, E.; Juusola, J.; Gilissen, C.; Baralle, D.; Reijnders, M.R.F.; Venselaar, H.; Helsmoortel, C.; Cho, M.T.; Hoischen, A.; et al. Mutations in DDX3X are a common cause of unexplained intellectual disability with gender-specific effects on wnt signaling. Am. J. Hum. Genet. 2015, 97, 343-352. [CrossRef]

23. Wang, X.; Posey, J.E.; Rosenfeld, J.A.; Bacino, C.A.; Scaglia, F.; Immken, L.D.; Harris, J.M.; Hickey, S.E.; Mosher, T.M.; Slavotinek, A.; et al. Phenotypic expansion in DDX3X-A common cause of intellectual disability in females. Ann. Clin. Transl. Neurol. 2018, 15, 1277-1285. [CrossRef]

24. Gilling, M.; Rasmussen, H.B.; Calloe, K.; Sequeira, A.F.; Baretto, M.; Oliveira, G.; Almeida, J.; Lauritsen, M.B.; Ullmann, R.; Boonen, S.E.; et al. Dysfunction of the heteromeric KV7.3/KV7.5 potassium channel is associated with autism spectrum disorders. Front. Genet. 2013, 4, 54. [CrossRef]

25. Nemani, T.; Steel, D.; Kaliakatsos, M.; DeVile, C.; Ververi, A.; Scott, R.; Getov, S.; Sudhakar, S.; Male, A.; Mankad, K.; et al. KIF1A-related disorders in children: A wide spectrum of central and peripheral nervous system involvement. J. Peripher. Nerv. Syst. 2020, 25, 117-124. [CrossRef]

26. Garde, A.; Cornaton, J.; Sorlin, A.; Moutton, S.; Nicolas, C.; Juif, C.; Geneviève, D.; Perrin, L.; Khau-Van-Kien, P.; Smol, T.; et al. Neuropsychological study in 19 French patients with White-Sutton syndrome and POGZ mutations. Clin. Genet. 2021. [CrossRef] 
27. Assia Batzir, N.; Posey, J.E.; Song, X.; Akdemir, Z.C.; Rosenfeld, J.A.; Brown, C.W.; Chen, E.; Holtrop, S.G.; Mizerik, E.; Nieto Moreno, M.; et al. Phenotypic expansion of POGZ-related intellectual disability syndrome (White-Sutton syndrome). Am. J. Med. Genet. Part A 2020, 182, 38-52. [CrossRef] [PubMed]

28. Precht, K.S.; Lese, C.M.; Spiro, R.P.; Huttenlocher, P.R.; Johnston, K.M.; Baker, J.C.; Christian, S.L.; Kittikamron, K.; Ledbetter, D.H. Two 22q telomere deletions serendipitously detected by FISH. J. Med. Genet. 1998, 35, 939-942. [CrossRef] [PubMed]

29. Durand, C.M.; Betancur, C.; Boeckers, T.M.; Bockmann, J.; Chaste, P.; Fauchereau, F.; Nygren, G.; Rastam, M.; Gillberg, I.C.; Anckarsäter, H.; et al. Mutations in the gene encoding the synaptic scaffolding protein SHANK3 are associated with autism spectrum disorders. Nat. Genet. 2007, 39, 25-27. [CrossRef] [PubMed]

30. Prasad, C.; Prasad, A.N.; Chodirker, B.N.; Lee, C.; Dawson, A.K.; Jocelyn, L.J.; Chudley, A.E. Genetic evaluation of pervasive developmental disorders: The terminal 22q13 deletion syndrome may represent a recognizable phenotype. Clin. Genet. 2000, 57, 103-109. [CrossRef] [PubMed]

31. Powers, P.A.; Scherer, S.W.; Tsui, L.C.; Gregg, R.G.; Hogan, K. Localization of the Gene Encoding the $\alpha^{2} / \delta$ Subunit (CACNL2A) of the Human Skeletal Muscle Voltage-Dependent $\mathrm{Ca}^{2+}$ Channel to Chromosome 7q21-q22 by Somatic Cell Hybrid Analysis. Genomics 1994, 19, 192-193. [CrossRef]

32. Vergult, S.; Dheedene, A.; Meurs, A.; Faes, F.; Isidor, B.; Janssens, S.; Gautier, A.; Le Caignec, C.; Menten, B. Genomic aberrations of the CACNA2D1 gene in three patients with epilepsy and intellectual disability. Eur. J. Hum. Genet. 2015, 23, 628-632. [CrossRef] [PubMed]

33. Bian, F.; Li, Z.; Offord, J.; Davis, M.D.; McCormick, J.; Taylor, C.P.; Walker, L.C. Calcium channel alpha2-delta type 1 subunit is the major binding protein for pregabalin in neocortex, hippocampus, amygdala, and spinal cord: An ex vivo autoradiographic study in alpha2-delta type 1 genetically modified mice. Brain Res. 2006, 1075, 68-80. [CrossRef] [PubMed]

34. Mori, M.; Sugo, T.; Abe, M.; Shimomura, Y.; Kurihara, M.; Kitada, C.; Kikuchi, K.; Shintani, Y.; Kurokawa, T.; Onda, H.; et al. Urotensin II is the endogenous ligand of a G-protein-coupled orphan receptor, SENR (GPR14). Biochem. Biophys. Res. Commun. 1999, 137, 579-588. [CrossRef] [PubMed] 